\title{
Cracking Condition of Cohesionless Porous Materials in Drying Processes
}

\author{
So Kitsunezaki* \\ Research Group of Physics, Division of Natural Sciences, \\ Faculty of Nara Women's University, Nara 630-8506, Japan
}

(Dated: November 4, 2018)

\begin{abstract}
The invasion of air into porous systems in drying processes is often localized in soft materials, such as colloidal suspensions and granular pastes, and it typically develops in the form of cracks before ordinary drying begins. To investigate such processes, we construct an invasion percolation model on a deformable lattice for cohesionless elastic systems, and with this model we derive the condition under which cracking occurs. A Griffith-like condition characterized by a dimensionless parameter is proposed, and its validity is checked numerically. This condition indicates that the ease with which cracking occurs increases as the particles composing the material become smaller, as the rigidity of the system increases, and as the degree of heterogeneity characterizing the drying processes decreases.

PACS numbers: 46.50.+a,64.70.fm,81.05.Rm,83.80.Hj
\end{abstract}

\section{INTRODUCTION}

Contraction that results from drying often causes the formation of cracks in pastelike materials such as wet granular materials and colloid suspensions. Compared with ordinary solids, such materials are soft in wet states due to weak cohesion among constituent particles, and they possess unique properties characterizing the crack formation that they exhibit, including memory effects, slow crack growth, and a diversity of crack patterns [112]. Considerable effort has been devoted to investigating the formation of crack patterns in the contexts of physics, soil mechanics, and geology, while cracking conditions have been investigated mainly in engineering applications.

During a drying process, the first crack in a paste generally appears in a capillary state, in which all pores (i.e., the spaces between solid particles) are filled with liquid, and the cracking process consists essentially of the invasion of air into these pores. We believe that cracking conditions can be deduced directly from material properties related to drying, because on the microscopic level, cracking is indistinguishable from ordinary drying in the elementary processes of air invasion. However, drying and cracking have been treated separately in previous theories, although some studies have investigated the effect of large deformation and the order of ordinary drying and cracking in drying processes 13 17].

The main goal of this paper is to determine the Griffith criterion associated with the drying process of cohesionless porous systems. For this purpose, we focus on the limit of slow drying in a uniform elastic system. The slow drying limit can be realized by decreasing the relative humidity $h$ quasistatically during the drying processes with fixed temperature $T$ and atmospheric pressure $P$. Under such conditions, both the water distribution and

\footnotetext{
* kitsune@ki-rin.phys.nara-wu.ac.jp http://www.complex.phys.nara-wu.ac.jp/ ${ }^{2}$ kitsune/main_e.html
}

elastic deformation of the material can be assumed to be in thermal equilibrium or quasiequilibrium. We also assume that the elastic relaxation is much faster than the redistribution of liquid, and we ignore plasticity, although it is believed to be important for many type of pastes [4, 7, 8, 11, 12, 18 20].

To investigate the systems of interest, we extend an invasion percolation (IP) model to include elastic interactions. It has been established that IP models are faithful models of drying processes in porous materials, despite the great simplification they employ of treating liquid distributions as binary distributions on a lattice [21 29]. Our extended model describes the cracklike invasion of air in soft systems with large rigidity. With it, we determine the condition for the formation of the first crack in terms of the free energy of the system. This condition is determined from the elastic properties of the material, the heterogeneity characterizing the drying processes, and the size of particles forming the paste.

In this paper, we investigated a two-dimensional system corresponding to a crosssection of a uniform layer of paste. We regard the bottom surface to be a fixed boundary and the top surface to be a free boundary and assume that the evaporation of liquid occurs only from the top surface. This paper is constructed as follows. We propose a theoretical model based on thermodynamic considerations in Sec. III. In Sec. III] we report the results of numerical simulations using this model. We study the conditions governing the invasion of air and the formation of the first crack in Sec. [V] and $\mathrm{V}$ ] respectively. Conclusions are given in Sec. VI.

\section{SLOW DESICCATION IN COHESIONLESS POROUS MATERIAL}

We regard the system as a mixture of solid particles and liquid forming a paste layer, and the environment as air containing vapor, which exists both inside and outside the paste layer. We treat this system and environment 
thermodynamically. In order to find the thermal equilibrium state for a given $h$ with fixed $(T, P)$, we introduce a free energy $J \equiv F+P\left(V_{s}+V_{l}\right)-\mu_{v}(T, P, h) N_{l}$, where $F$, $V_{s}, \mu_{v}, N_{l}$, and $V_{l}$ are the Helmholtz free energy, the total volume of the constituent particles, the chemical potential of the vapor, the number of liquid molecules, and the volume of the liquid, respectively. We ignore the effect of gravity and assume that $V_{s}$ and $V_{l} / N_{l}$ are constant.

The Helmholtz free energy, $F$, is given by the sum $F \equiv F_{e}+F_{i}+F_{l}$. Here, $F_{e}$ represents the deformation energy of the system, which results from interactions among particles. The surface energy, $F_{i}$, is determined by the liquid distribution and increases as the invasion of air progresses, as shown in Appendix $\mathrm{A}$. The free energy of the liquid, $F_{l}$, is given by $F_{l}=-P_{l} V_{l}+\mu_{l} N_{l}$, as obtained from the Gibbs-Dühem relation, where $P_{l}$ is the hydrostatic pressure of the liquid, and the chemical potential of the liquid, $\mu_{l}$, is identical to $\mu_{v}$. Substituting these forms into the above expression for $J$, and defining $p \equiv P-P_{l}$, we obtain

$$
J \equiv F_{e}+F_{i}+p(T, P, h) V_{l}+\text { const. }
$$

The equilibrium state of the paste for given $h$ is determined by minimizing $J$ with respect to the other state variables.

The difference between the hydrostatic pressure of the fluid in the pores and the atmospheric pressure, $p$, is called the negative pore pressure in soil mechanics. The pressure $p$ is uniform throughout the system in equilibrium states and determined uniquely by $h$ for given $T$ and $P$, in accordance with the Kelvin equation, as indicated in Appendix A We adopt $p$ as the control parameter in place of $h$, because $p$ corresponds to the driving force of air invasion. In the processes we consider, $p$ increases in time, while $h$ decreases as a function of $p$.

Here we give a brief remark concerning the minimization of $J$ for processes occurring at fixed $p$. First, we note that even when $p$ increases quasistatically, a region into which air has invaded often expands abruptly, exhibiting behavior similar to that in an avalanche process. However, if the redistribution and desiccation of liquid are sufficiently slow, such a process can still be regarded as proceeding slowly, and, therefore, we can assume that the system is approximately in mechanical equilibrium throughout. While $V_{l}$ decreases gradually, instantaneous equilibrium states (characterized by instantaneous values of $V_{l}$ ) can be obtained by minimizing $F$. Technically, this implies that we can always use the minimum principle of $J$ by including $V_{l}$ into the set of control parameters during such a process, because the difference between $J$ and $F, P\left(V_{s}+V_{l}\right)-\mu_{v}(T, P, h(T, P, p)) N_{l}$, is constant if $V_{l}$ is fixed, in addition to $(T, P, p)$.

\section{A. Rigid materials}

We use a regular lattice composed of $M$ cells each of volume $\Delta V$ to represent the system. The liquid distri- bution on the lattice is described by the set of variables $\left\{\phi_{1}, \cdots, \phi_{M}\right\}$, where $\phi_{m}$ represents the "dryness" of the $m$ th cell. Each cell is assumed to be either wet $\left(\phi_{m}=0\right)$ or dry $\left(\phi_{m}=1\right)$, as in percolation models. In order for such a treatment to be valid, we must assume that the cells possess microscopic volumes. We adopt the length of a cell as the unit of length in our model, which is proportional to the linear extent of a particle, $r$, fixing all other microscopic properties. In addition, we assume that all wet cells have the same liquid volume fraction, $v_{w}$, and that no liquid exists in dry cells, for simplicity. The volume occupied by solid particles in each cell is $\left(1-v_{w}\right) \Delta V$.

The total volume of liquid and surface energy are

$$
V_{l} \equiv \sum_{m=1}^{M} \Delta V v_{w}\left(1-\phi_{m}\right),
$$

and

$$
F_{i} \equiv \gamma_{l a} \sum_{m=1}^{M} \Delta A_{m} \phi_{m}+\text { const. }
$$

respectively, where $\gamma_{l a}$ is the surface tension of the liquidair interface. The quantity $\gamma_{l a} \Delta A_{m}$ is the increase in the surface energy needed to change the state of the $m$ th cell from wet to dry. This represents the resistance to drying. In order to investigate heterogeneous material, we assume that $\Delta A_{m}$ varies among the cells, with an average value $\overline{\Delta A}$. The characteristic pressure in a drying process,

$$
p_{\gamma} \equiv \gamma_{l a} \frac{\overline{\Delta A}}{v_{w} \Delta V} \sim \frac{\gamma_{l a}}{r},
$$

is typically on the order of the surface tension divided by $r$.

Because $F_{e}$ is constant for rigid systems, we have

$$
J=\sum_{m=1}^{M}\left[\gamma_{l a} \Delta A_{m} \phi_{m}+p v_{w}\left(1-\phi_{m}\right) \Delta V\right]+\text { const. }
$$

Introducing the quantities $\tilde{J} \equiv J / v_{w} p_{\gamma}, \gamma_{m} \equiv \Delta A_{m} / \overline{\Delta A}$, and $\tilde{p} \equiv p / p_{\gamma}$, this expression can be written in the simplified form

$$
\tilde{J}=\sum_{m=1}^{M} \Delta V\left\{\gamma_{m} \phi_{m}+\tilde{p}\left(1-\phi_{m}\right)\right\},
$$

after removing the constant from $J$.

This free energy is minimized when the liquid distribution satisfies the conditions

$$
\left\{\begin{array}{l}
\phi_{m}=0 \text { for } \gamma_{m}>\tilde{p} \\
\phi_{m}=1 \text { for } \gamma_{m} \leq \tilde{p}
\end{array} .\right.
$$

If these conditions are applied only to cells adjacent to the dry-wet interface, this process corresponds to that described by the conventional IP model without a trapping 
rule $[21-23]$. In the present work, we assume that $\gamma_{m}$ is distributed uniformly over the interval $[1-\Delta \gamma, 1+\Delta \gamma]$ and that $\Delta \gamma \ll 1$. For large systems, air invades when $\tilde{p} \simeq 1-\Delta \gamma$ from the drying surface, and it percolates above a certain threshold $\tilde{p}=p_{c}<1+\Delta \gamma$, as is well known.

Here, we note that the change of a state from wet to dry is reversible and may be caused simply by the redistribution of liquid, rather than desiccation, as observed by $\mathrm{L}$. $\mathrm{Xu}$ et al. [30]. Also, desiccation in the vicinity of the free surface generally induces liquid flow which causes the invasion of air far from this surface. Such flows are often maintained in wet regions with high resistance to drying and facilitate the drying processes [31].

\section{B. Extension to elastic materials}

We regard each cell as an elastic tile subject to uniform strains, in order to describe the deformation of the system. The elastic energy $F_{e}$ is determined by the strains as $F_{e}\left\{\boldsymbol{U}_{m}\right\} \equiv \sum_{m=1}^{M} \Delta V f_{e}\left(\boldsymbol{U}_{m}\right)$, where $\boldsymbol{U}_{m} \equiv\left(u_{\alpha \beta}^{(m)}\right)$ is the strain tensor of the $m$ th cell and $f_{e}$ is the free energy density. In order to simplify the situation, we make the following assumptions: (a1) all cells have the same elastic properties, (a2) $f_{e}$ does not depend on $\phi_{m}$, and (a3) $\Delta A_{m}$ does not depend on $\boldsymbol{U}_{m}$. Heterogeneity is introduced into the system only through the drying process.

Coupling of $\phi_{m}$ and $\boldsymbol{U}_{m}$ is introduced through $V_{l}$. The dilation of a cell results from the influx of liquid for a wet cell and an influx of air for a dry cell. To account for such phenomena, we add the volumetric strain $u_{\alpha \alpha}^{(m)}$ to $v_{w}$ in Eq. (2). This yields the free energy

$$
\begin{aligned}
J= & F_{e}\left\{\boldsymbol{U}_{m}\right\}+\sum_{m=1}^{M}\left[\gamma_{l a} \Delta A_{m} \phi_{m}\right. \\
& \left.+p\left(v_{w}+u_{\alpha \alpha}^{(m)}\right)\left(1-\phi_{m}\right) \Delta V\right]+ \text { const },
\end{aligned}
$$

where Einstein's summation rule is applied to repeated Greek indices. Then, introducing $\tilde{\boldsymbol{U}}_{m} \equiv \boldsymbol{U}_{m} / v_{w}$ and $\tilde{f}_{e}\left(\tilde{\boldsymbol{U}}_{m}\right) \equiv f_{e}\left(\boldsymbol{U}_{m}\right) / v_{w} p_{\gamma}$, we obtain

$$
\tilde{J}=\sum_{m=1}^{M} \Delta V\left\{\tilde{f}_{e}\left(\tilde{\boldsymbol{U}}_{m}\right)+\gamma_{m} \phi_{m}+\tilde{p}\left(1+\tilde{u}_{\alpha \alpha}^{(m)}\right)\left(1-\phi_{m}\right)\right\} .
$$

We note that the assumption (a3) can be weakened slightly in the case that $\Delta A_{m}$ depends on $\boldsymbol{U}_{m}$ linearly as $\Delta A_{m}=\Delta \bar{A}\left(\gamma_{m}+\gamma^{\prime} \tilde{u}_{\alpha \alpha}^{(m)}\right)$, where $\gamma^{\prime}$ is a constant, because an expression identical to Eq. (9) can be obtained through an appropriate transformation of the variables.

With the elastic energy included, the conditions to determine the liquid distribution are revised from those appearing in Eq. (7) to

$$
\left\{\begin{array}{l}
\phi_{m}=0 \text { for } \gamma_{m}>\tilde{p}\left(1+\tilde{u}_{\alpha \alpha}^{(m)}\right) \\
\phi_{m}=1 \text { for } \gamma_{m} \leq \tilde{p}\left(1+\tilde{u}_{\alpha \alpha}^{(m)}\right)
\end{array} .\right.
$$

These conditions imply that the resistance to drying decreases as a cell expands, because the expansion of a cell decreases the cost in surface energy required to remove a unit volume of liquid.

The free energy has a minimum with respect to $\left\{\tilde{\boldsymbol{U}}_{m}\right\}$ in the equilibrium state. Minimizing Eq. (9) in the continuum limit gives the stress balance equation

$$
\frac{\partial \tilde{\sigma}_{\alpha \beta}}{\partial x_{\beta}}=0,
$$

where $x_{\beta}$ represents the space coordinates and the stresses $\tilde{\sigma}_{\alpha \beta}$ are defined by

$$
\tilde{\sigma}_{\alpha \beta} \equiv \frac{\partial \tilde{f}_{e}}{\partial \tilde{u}_{\alpha \beta}}+\tilde{p}(1-\phi) \delta_{\alpha \beta} .
$$

Solid particles in wet regions are subject to compressive pressure $\tilde{p}$ from both the free surface and the interface with dry regions.

\section{Elastic energy of cohesionless materials}

Soft materials that exhibit drying cracks, such as colloid suspensions and wet granular materials, generally have nonlinear elastic properties. In many cases, the cohesive interactions of the constituent particles are very weak in comparison with excluded volume interactions, and materials in capillary states hold their shape under compressive stresses caused by negative pore pressures. 32] The elastic energy and moduli practically vanish unless the system is subject to compressive stresses. Therefore, we assume that $\tilde{f}_{e}(\tilde{\boldsymbol{U}})=0$ for $\tilde{u}_{\alpha \alpha} \geq 0$ and that the elastic moduli vanish for $\tilde{u}_{\alpha \alpha}=0$.

We need to choose an appropriate function of $\tilde{f}_{e}(\tilde{\boldsymbol{U}})$ for $\tilde{u}_{\alpha \alpha}<0$, because there is no established general constituent relation. If we assume an isotropic analytic function for the elastic moduli, $\tilde{f}_{e}$ can be approximated in the form of a third-order elasticity as $\tilde{f}_{e}=$ $-\lambda \tilde{u}_{\alpha \alpha}^{3}-\mu \tilde{u}_{\xi \xi} \tilde{u}_{\alpha \beta}^{2}$ for small $\tilde{u}_{\alpha \alpha}$, where $\lambda$ and $\mu$ are positive constants. L. Goehring reported that this thirdorder elasticity accurately describes the results of compression tests with cornstarch paste [33]. Another choice is $\tilde{f}_{e}=\sqrt{-\tilde{u}_{\xi \xi}}\left(\lambda \tilde{u}_{\alpha \alpha}^{2}+\mu \tilde{u}_{\alpha \beta}^{2}\right)$, which is obtained theoretically by assuming Hertzian contacts among particles and affine deformation [16, 34].

We assume the following general homogeneous form including these choices:

$$
\tilde{f}_{e}(\tilde{\boldsymbol{U}})=g\left(\tilde{u}_{\xi \xi}\right)\left[\frac{1}{2} \tilde{K} \tilde{u}_{\alpha \alpha}^{2}+\tilde{G}\left(\tilde{u}_{\alpha \beta}-\frac{1}{2} \tilde{u}_{\eta \eta} \delta_{\alpha \beta}\right)^{2}\right],
$$

where $\tilde{K}$ and $\tilde{G}$ are positive constants. The function $g(x)$ takes the power-law form

$$
g(x)=\left\{\begin{array}{ll}
(-x)^{\nu-1} & x<0 \\
0 & x \geq 0
\end{array},\right.
$$




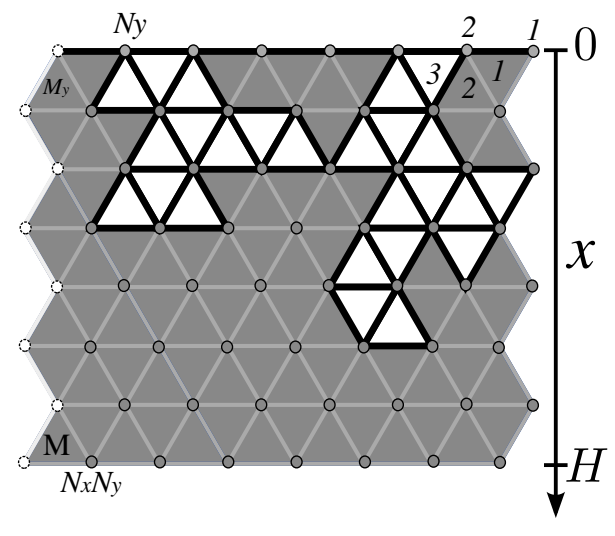

FIG. 1. Triangular lattice with $N_{x} \times N_{y}$ vertices and $M=$ $2 N_{y}\left(N_{x}-1\right)$ cells. The $x$-axis is normal to the surfaces of the system, with the value of $x$ representing the distance from the top surface. Dry cells are enclosed by bold lines.

where $\nu=2$ for third-order elasticity and $\nu=3 / 2$ in the case of Hertzian contacts. The bulk modulus and rigidity depend on the state of the material, due to the nonlinearity. As shown in Appendix B, they are proportional to $g\left(\tilde{u}_{\alpha \alpha}\right)$ for isotropic compressive states, and $2 \tilde{G}<\nu(\nu+1) \tilde{K}$ is required for most materials with positive Poisson's ratio. We investigated our model mainly for large $\tilde{K}$, as Eq. (13) generally holds for small deformations.

\section{NUMERICAL SIMULATIONS}

\section{A. Methods}

We consider a uniform layer from whose top surface (which is a one-dimensional interface) liquid is desiccated. We carried out numerical simulations using thirdorder elasticity $(\nu=2)$ on a two-dimensional triangular lattice, as shown in Fig. 1. The lattice has $N_{x} \times N_{y}$ vertices, and the layer thickness is $H \equiv \sqrt{3}\left(N_{x}-1\right) / 2$.

In order to avoid erroneous numerical convergence due to the singularity at $\tilde{u}_{\alpha \alpha}=0$ for $g^{\prime}(x)$, we used the smooth function

$$
g(x)= \begin{cases}-x & \text { for } b x<-1 \\ \frac{1}{4 b}(b x-1)^{2} & \text { for }|b x| \leq 1 \\ 0 & \text { for } b x>1\end{cases}
$$

with a large positive constant $b=10^{4}$, instead of the form given in Eq. (14).

The numerical method we used is essentially the same as that used in Ref. [35]. The deformation of the lattice is described by the displacements of the vertices, $\left\{\boldsymbol{u}_{1}, \cdots, \boldsymbol{u}_{N}\right\}\left(N \equiv N_{x} N_{y}\right)$, which determine $\left\{\tilde{\boldsymbol{U}}_{m}\right\}$. The top surface is a free boundary in contact with air. The interface between air and wet cells is treated as the dry-wet interface. The bottom surface is a fixed boundary with respect to $\left\{\boldsymbol{u}_{n}\right\}$ and a reflecting boundary with respect

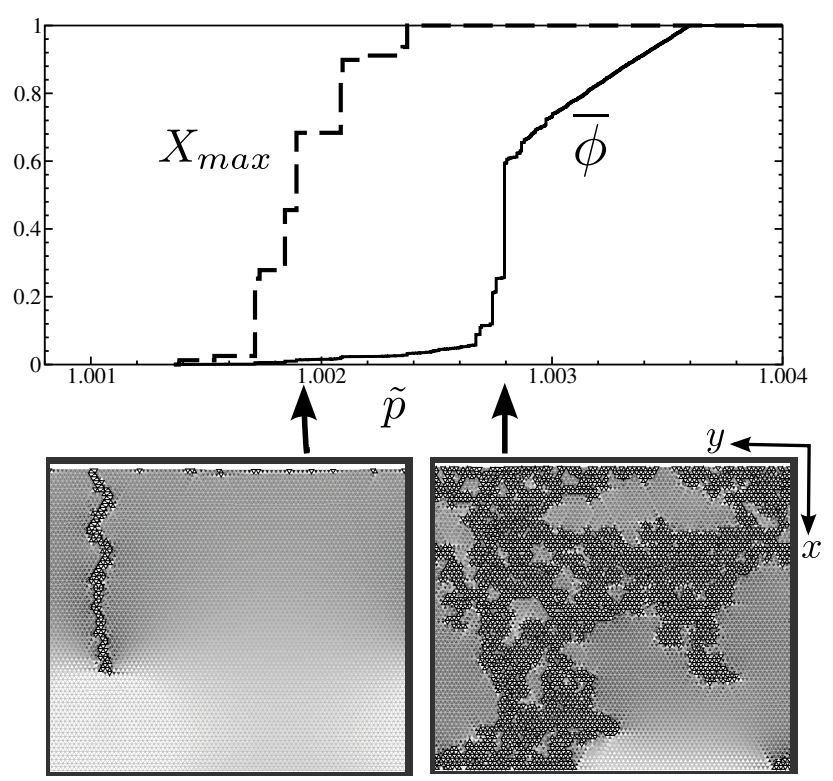

FIG. 2. The drying process following the cracklike invasion of air. The graph displays the results for the dry fraction $\bar{\phi}$ and the maximum depth of a dry cell, $X_{\max }$, as functions of $\tilde{p}$ obtained from a numerical simulation with $(\tilde{K}, \tilde{G})=$ $\left(10^{5}, 2 \times 10^{4}\right), \Delta \gamma=0.001$, and $N_{x}=N_{y}=80$. The bottom snapshots correspond to $(\tilde{p}-1) / \Delta \gamma=1.19$ and 2.79 .

to $\left\{\phi_{m}\right\}$. Periodic boundary conditions are used along the $y$ direction. The following procedures were repeated in the numerical simulations from the initial conditions in which all cells were wet and undeformed.

(P1): The displacements $\left\{\boldsymbol{u}_{n}\right\}$ were calculated by minimizing $\tilde{J}$ for fixed $\left\{\phi_{m}\right\}$ and $\tilde{p}$ in Eqs. (9) and (13) using the conjugate gradient method [36].

(P2): The conditions (10) were checked for all cells contacting the dry-wet interface. If these conditions were satisfied, we increased $\tilde{p}$ by $\Delta \tilde{p}$ and then returned to $(\mathrm{P} 1)$.

(P3): If the $m$ th cell did not satisfy these conditions, we changed $\phi_{m}$ from wet (dry) to dry (wet) and returned to $(\mathrm{P} 1)$.

In (P3), if we found more than one cell that did not satisfy the conditions (10), we changed only the state of the most unstable cell, i.e., that with the largest deviation from the condition $\gamma_{m}=\tilde{p}\left(1+\tilde{u}_{\alpha \alpha}^{(m)}\right)$. Under this procedure, $V_{l}$ changes gradually for fixed $\tilde{p}$. In the simulations whose results are presented here, we used $\Delta \tilde{p}=\Delta \gamma / 500$, and the tolerance of the conjugate gradient method was $3 \times 10^{-11}$. The numerical results were confirmed to be essentially the same with those obtained for smaller $\Delta \tilde{p}$ and tolerance. 


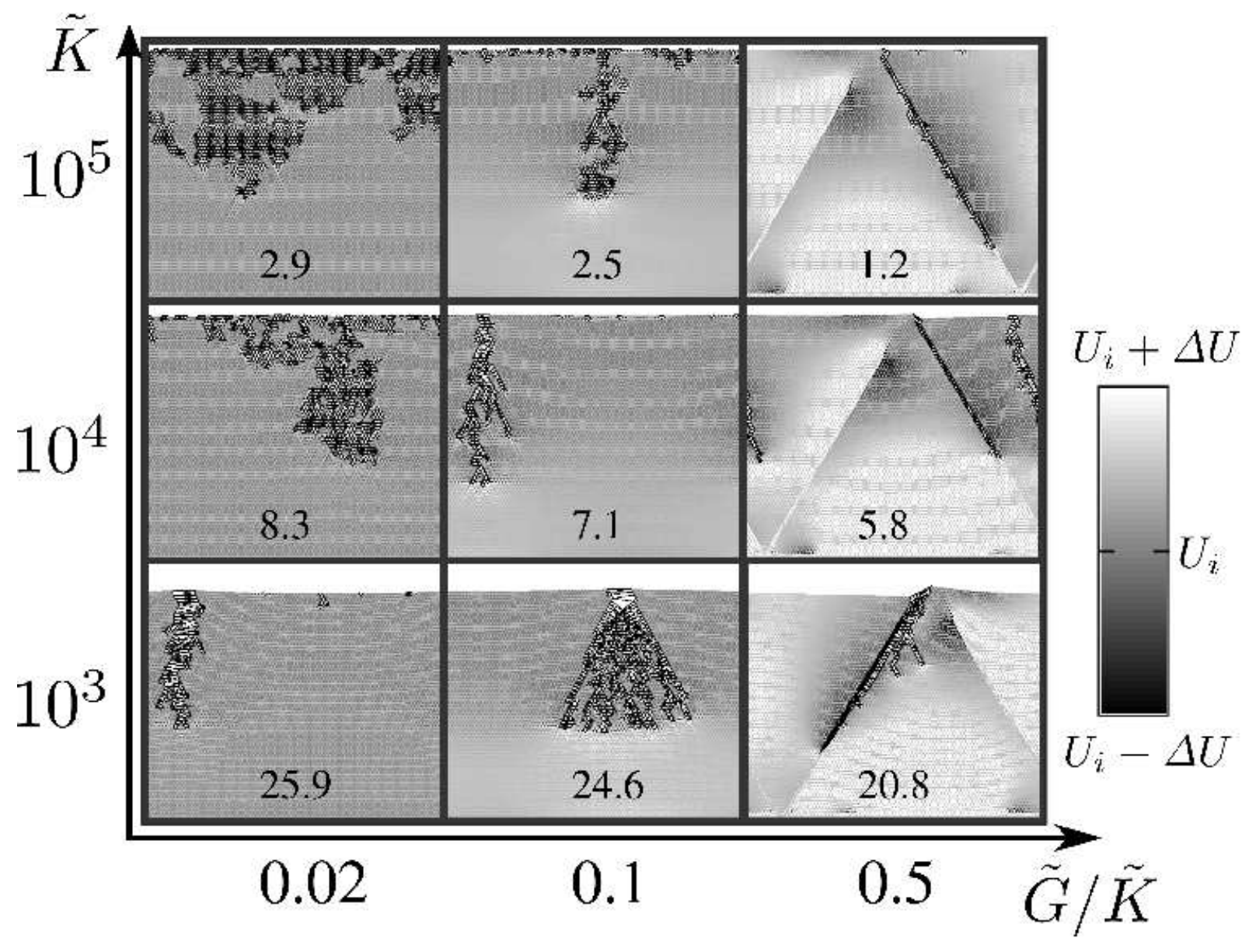

FIG. 3. Snapshots of air invasion for $\Delta \gamma=0.001, \tilde{K}=10^{3}, 10^{4}, 10^{5}$ and $\tilde{G} / \tilde{K}=0.02,0.1,0.5$. In all cases, $N_{x}=N_{y}=80$. The number appearing in each figure indicates the value of $(\tilde{p}-1) / \Delta \gamma$ for that snapshot. The deformation of the lattice is scaled by a factor of 5 for viewability, and the volumetric strain $\tilde{u}_{\alpha \alpha}$ is indicated by the gray scale, which corresponds to the interval $\left[\tilde{U}_{i}-\Delta U, \tilde{U}_{i}+\Delta U\right]$, where $\tilde{U}_{i}$ is given by Eq. (20) and $\Delta U=1 /(6 \sqrt{10 \tilde{K}})$.

\section{B. Results}

Our numerical results indicate that the cracklike invasion of air occurs readily in soft systems with large rigidity and small heterogeneity.

Figure 2 depicts a typical process of cracklike invasion and subsequent drying. As soon as a cell on the top surface dries, air penetrates rapidly into the bulk and this results in the formation of a one-dimensional dry region. This dry region expands gradually from both the top surface and the crack line as $\tilde{p}$ increases. The graph in this figure plots the fraction of the entire system in the dry region, $\bar{\phi} \equiv \sum_{m=1}^{M} \phi_{m} / M$, and the maximum depth of a dry cell divided by the thickness, $X_{\text {max }} \equiv \max _{m}\left\{x_{m} \mid \phi_{m}=1\right\} / H$, as functions of $\tilde{p}$. It is found that soft material resists the invasion of air through shrinkage. When shrinkage occurs, the pressure required for air invasion to begin is larger than that in the case of the conventional IP model, $\tilde{p} \simeq 1-\Delta \gamma$. On the other hand, the percolation threshold decreases drastically if the cracklike invasion of air occurs.
Figure 3 displays typical snapshots for nine sets of $(\tilde{K}, \tilde{G})$ and fixed $\Delta \gamma$. These were taken after a dry region had developed beyond a depth of approximately half of the total depth. For sufficiently large $\tilde{K}$ or small $\tilde{G}$, the air invasion process occurs in the same manner as for the conventional IP model. It always occurs at $\tilde{G}=0$ for any value of $\tilde{K}$, as described below. The dry region expands intermittently and gradually as the pressure $\tilde{p}$ increases. Contrastingly, for large $\tilde{G}$ and small $\tilde{K}$, cracklike air invasion occurs first. This dry region essentially corresponds to a mode I crack, because the cells in this region are expanded (i.e., $\tilde{u}_{\alpha \alpha}>0$ ), while the surrounding wet region shrinks. For sufficiently large $\tilde{G}$ and small $\tilde{K}$, however, shear bands (mode II cracks) often form ahead of cracks in the wet region. The directions of the shear bands reflect the anisotropy of the triangular lattice. The wet cells in such shear bands expand until $\tilde{u}_{\alpha \alpha} \simeq 0$, and cracklike air invasion develops along shear bands. Shear bands sometimes appear and disappear during the invasion of air.

Figure 4 elucidates the dependence of the heterogene- 
ity for fixed $(\tilde{K}, \tilde{G})$. As $\Delta \gamma$ increases, the air invasion process changes from cracklike to conventional IP-like. The value of $\Delta \gamma$ at which this change occurs depends on $(\tilde{K}, \tilde{G})$, as discussed in Sec. D

The transition between cracklike and conventional IPlike invasion has been reported for some heterogeneous systems with long-range interactions [37, 38]. In particular, R. Holtzman et al. investigated the displacement of immiscible fluid in preloaded granular systems and reported three types of invasion: fracturing, capillary fingering $(\mathrm{CF})$, and viscous fingering $(\mathrm{VF})$ 39, 40]. Fracturing and $\mathrm{CF}$ in their systems correspond to cracklike and conventional IP-like invasions, respectively, although loading arises from increasing negative pore pressures in our systems. VF is caused by the effect of pressure gradient. Although it does not occur in the slow drying limit, the gradient of negative pore pressures becomes important for fast drying and results in directional cracking generally [11, 41].

\section{INVASION OF AIR}

When the invasion of air does not occur yet, the system is uniform and contracts only along the $x$ direction as $\tilde{p}$ increases with drying. Such uniaxial compressive states are described by $\phi=0$ and

$$
\left(\tilde{u}_{\alpha \beta}\right)=\left(\begin{array}{cc}
\tilde{U}_{a} & 0 \\
0 & 0
\end{array}\right) \equiv \tilde{\boldsymbol{U}}_{a},
$$

where $\tilde{U}_{a}$ is determined from the free boundary condition $\tilde{\sigma}_{x x}=\partial \tilde{f}_{e} / \partial \tilde{U}_{a}+\tilde{p}=0$ on the top line. Substituting these values into Eqs. (13) and (14), we obtain $\tilde{f}_{e}=$ $(\tilde{K}+\tilde{G})\left(-\tilde{U}_{a}\right)^{\nu+1} / 2$ and then

$\tilde{U}_{a}=-\left\{\frac{2 \tilde{p}}{(\nu+1)(\tilde{K}+\tilde{G})}\right\}^{\frac{1}{\nu}}$ and $\tilde{f}_{e}\left(\tilde{\boldsymbol{U}}_{a}\right)=-\frac{1}{\nu+1} \tilde{p} \tilde{U}_{a}$.

If the top surface is sufficiently long, the invasion of air begins when the second condition in (10) holds at a cell on the surface for which we have

$$
1-\Delta \gamma=\tilde{p}\left(1+\tilde{U}_{a}\right),
$$

because the smallest $\gamma_{m}$ is $1-\Delta \gamma$. The pressure at which the invasion of air begins is determined by this condition and increases as $\tilde{K}+\tilde{G}$ decreases. Because the right-hand side of Eq. (18) has a maximum at $\tilde{U}_{a}=-\nu /(\nu+1)$, the invasion of air never occurs for systems so soft that the condition

$$
\tilde{K}+\tilde{G}<2\left(1+\frac{1}{\nu}\right)^{\nu}(1-\Delta \gamma) \simeq 2\left(1+\frac{1}{\nu}\right)^{\nu}
$$

holds. This case corresponds to wet sintering in this system [1, 13 16].
Let us first consider a drying process for $\tilde{G}=0$, that is, the case with no rigidity. In this case, the system does not exhibit cracking and dries in the same manner as in the conventional IP model. We can solve the stress balance equation (11) easily in the case $\tilde{G}=0$, and we obtain isotropic stress states with $\tilde{\sigma}_{\alpha \beta}=0$ and

$$
\tilde{u}_{\alpha \alpha}=-\left\{\frac{2 \tilde{p}}{(\nu+1) \tilde{K}}\right\}^{\frac{1}{\nu}} \equiv \tilde{U}_{i}
$$

for all wet cells and $\tilde{u}_{\alpha \alpha} \geq 0$ for dry cells. The elastic energy of a wet cell is the same as that of a cell in the isotropic compressive state,

$$
\tilde{f}_{e}\left(\tilde{\boldsymbol{U}}_{i}\right)=-\frac{1}{\nu+1} \tilde{p} \tilde{U}_{i}, \text { where } \tilde{\boldsymbol{U}}_{i} \equiv \frac{\tilde{U}_{i}}{2}\left(\begin{array}{ll}
1 & 0 \\
0 & 1
\end{array}\right) .
$$

The pressure at which the $m$ th cell is allowed to dry is determined from $\gamma_{m}=\tilde{p}\left[1+\tilde{U}_{i}(\tilde{p})\right]$, in accordance with the conditions in (10), along with the condition $\tilde{U}_{i}>-\nu /(\nu+1)$. Because the pressure always increases monotonically as $\gamma_{m}$ increases, the order of drying does not depend on $\tilde{K}$.

The above conclusion concerning the nature of the drying can also be obtained by considering the free energy, $J$. To show this, we first note that additional energy is required to dry cells on an elastic lattice, because the elastic energy stored in a cell is dissipated quickly through dilation following drying. In order to develop a dry region $D$ for a fixed value of $\tilde{p}$, the amount by which the free energy $\tilde{J}$ decreases must be larger than the dissipation,

$$
R \equiv \sum_{m \in D} \Delta V \tilde{f}_{e}\left(\tilde{\boldsymbol{U}}_{m}^{(\mathrm{dry})}\right),
$$

where $\tilde{\boldsymbol{U}}_{m}^{(\mathrm{dry})}$ is the strain on the $m$ th cell at the time that it changes from wet to dry. This condition can be written

$$
\tilde{J}(\tilde{p} ; \emptyset)-\tilde{J}(\tilde{p} ; D)-R=\sum_{m \in D} \Delta V\left(\tilde{p}\left(1+\tilde{U}_{i}\right)-\gamma_{m}\right)>0,
$$

because $f_{e}\left(\tilde{\boldsymbol{U}}_{m}^{\text {(dry) }}\right)=f_{e}\left(\tilde{\boldsymbol{U}}_{i}\right)$, and Eq. (9) can be rewritten as $\tilde{J}(\tilde{p} ; D)=\sum_{m \notin D} \Delta V\left\{\tilde{f}_{e}\left(\tilde{\boldsymbol{U}}_{i}\right)+\tilde{p}\left(1+\tilde{U}_{i}\right)\right\}+$ $\sum_{m \in D} \Delta V \gamma_{m}$, where $D=\emptyset$ (empty set) corresponds to the initial state, in which all cells are wet. When the invasion of air begins with the condition (18), the righthand side is negative, because $\tilde{p}\left(1+\tilde{U}_{i}\right)=1-\Delta \gamma<\gamma_{m}$. Therefore, the dry region can expand only in a step-bystep manner with increasing $\tilde{p}$, and, hence, there is no cracking.

\section{CRACKING}

\section{A. Fracture criterion for the first crack}

Next, we consider the case $\tilde{G} \neq 0$ and investigate the cracking condition. Cracking in our system consists of a 


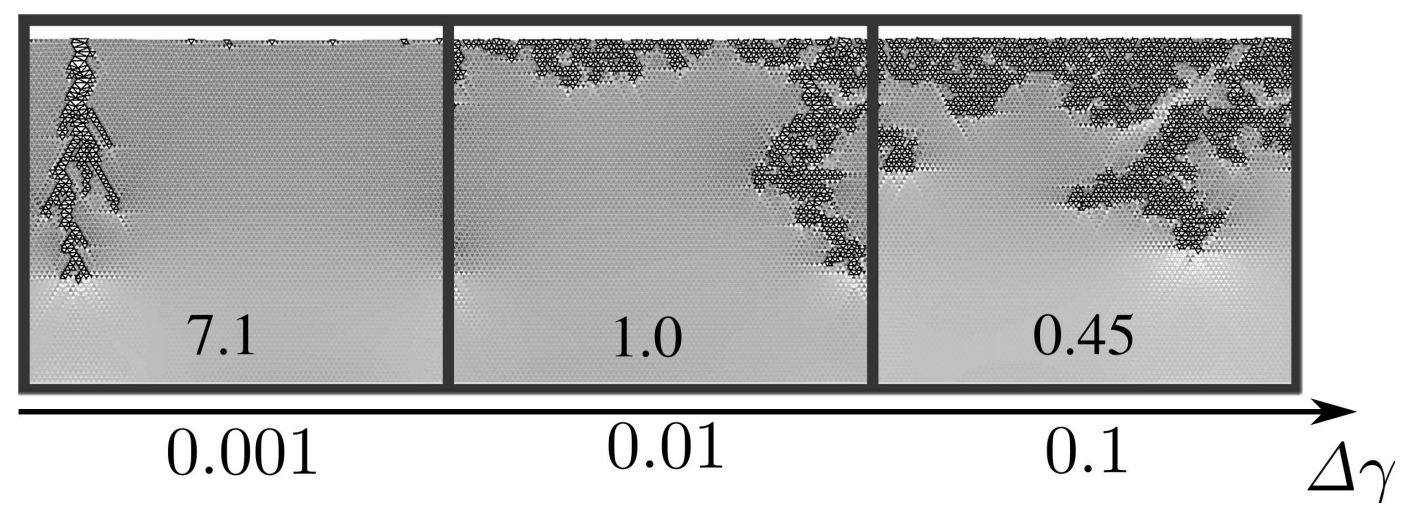

FIG. 4. Snapshots of air invasions for $\Delta \gamma=0.001,0.01,0.1$ and fixed $(\tilde{K}, \tilde{G})=\left(10^{4}, 10^{3}\right)$. These snapshots are displayed in the same manner as those in Fig. 3. In all cases, $N_{x}=N_{y}=80$. The number in each figure indicates the value of $(\tilde{p}-1) / \Delta \gamma$ for the respective values of $\Delta \gamma$.

quasi-one-dimensional invasion of air. It is facilitated by the release of energy from a surrounding wet region. Just as in the situation considered in the previous section, a crack develops if the amount by which the free energy $\tilde{J}$ decreases is larger than the dissipation $R$ when the invasion of air begins.

If there appears a crack of length $L$ that is perpendicular to the free surface in a sufficiently large system with fixed $\tilde{p}$, this crack forms an air-invaded region $D$ and it causes the elastic energy stored in $D$ to dissipate locally. The wet region $S$ surrounding the crack, from which the elastic energy is released, has an area of approximately $L \times L$, because $L$ is the only characteristic length in this system. The states in this region change from the uniaxial compressive state, characterized by $\tilde{\boldsymbol{U}}_{a}$, to an approximately isotropic compressive state, characterized by $\tilde{\boldsymbol{U}}_{i}$. Measuring $L$ by the number of cells in $D$, the decrease of $\tilde{J}$ is estimated as

$$
\begin{aligned}
\tilde{J}(\tilde{p} ; \emptyset) & -\tilde{J}(\tilde{p} ; D) \\
& \simeq L^{2} \Delta V\left\{\tilde{f}_{e}\left(\tilde{\boldsymbol{U}}_{a}\right)-\tilde{f}_{e}\left(\tilde{\boldsymbol{U}}_{i}\right)+\tilde{p}\left(\tilde{U}_{a}-\tilde{U}_{i}\right)\right\} \\
& +L \Delta V\left\{\tilde{f}_{e}\left(\tilde{\boldsymbol{U}}_{a}\right)+\tilde{p}\left(1+\tilde{U}_{a}\right)-\gamma_{D}(L)\right\},
\end{aligned}
$$

where the average of $\gamma_{m}$ in $D$ is represented by

$$
\gamma_{D}(L) \equiv \frac{1}{L} \sum_{m \in D} \gamma_{m}
$$

The elastic energy of the $m$ th cell in $D, f_{e}\left(\tilde{\boldsymbol{U}}_{m}\right)$, dissipates with drying at $\gamma_{m} \simeq \tilde{p}\left(1+\tilde{u}_{\alpha \alpha}^{(m)}\right)$. A cell is deformed at a crack tip and expands by at least an amount $\tilde{u}_{\alpha \alpha}^{(m)}-\tilde{U}_{a} \simeq\left(\gamma_{m}-1+\Delta \gamma\right) / \tilde{p}=O(\Delta \gamma)$ in comparison with Eq. (18). However, as shown in Appendix C, the energy in this case is the same as $f_{e}\left(\tilde{\boldsymbol{U}}_{a}\right)$ to first order in $\Delta \gamma$, because the cell contacts a dry-wet interface. Thus, the minimum dissipation for air invasion is

$$
R=L \Delta V \tilde{f}_{e}\left(\tilde{\boldsymbol{U}}_{a}\right)+O\left((\Delta \gamma)^{2}\right) .
$$

The cracking condition $\tilde{J}(\tilde{p} ; \emptyset)-\tilde{J}(\tilde{p} ; D)-R>0$ for fixed $\tilde{p}$ and $D$ is approximated as

$$
\begin{aligned}
& L\left\{\tilde{f}_{e}\left(\tilde{\boldsymbol{U}}_{a}\right)-\tilde{f}_{e}\left(\tilde{\boldsymbol{U}}_{i}\right)+\tilde{p}\left(\tilde{U}_{a}-\tilde{U}_{i}\right)\right\} \\
& \gtrsim \gamma_{D}(L)-\tilde{p}\left(1+\tilde{U}_{a}\right)=\gamma_{D}(L)-1+\Delta \gamma,
\end{aligned}
$$

where we have used Eq. (18). The right-hand side of this expression is positive and order $\Delta \gamma$. The cracking condition of the system is determined by finding the minimum value of $\gamma_{D}(L)$ over all possible crack paths $D$. This yields

$$
L\left\{\tilde{f}_{e}\left(\tilde{\boldsymbol{U}}_{a}\right)-\tilde{f}_{e}\left(\tilde{\boldsymbol{U}}_{i}\right)+\tilde{p}\left(\tilde{U}_{a}-\tilde{U}_{i}\right)\right\} \gtrsim c(L) \Delta \gamma,
$$

where $c(L) \Delta \gamma \equiv \min _{D}\left(\gamma_{D}(L)-1+\Delta \gamma\right)$. The quantity $c(L)$ increases from $c(1) \simeq 0$ to a value less than 1 as $L$ increases.

The condition derived above corresponds to the Griffith criterion for the first crack in a drying process. It indicates that there is a critical crack length $L=L_{c}$ beyond which unstable crack growth occurs. The left-hand side of Eq. (28) is the energy released when the crack advances by one cell. The right-hand side, $c(L) \Delta \gamma$, is the additional energy required to dry a cell at a crack tip. The critical length, $L_{c}$, is determined by the nondimensional parameter

$$
\Gamma \equiv \Delta \gamma\left\{\tilde{f}_{e}\left(\boldsymbol{U}_{a}\right)-\tilde{f}_{e}\left(\tilde{\boldsymbol{U}}_{i}\right)+\tilde{p}\left(\tilde{U}_{a}-\tilde{U}_{i}\right)\right\}^{-1}
$$

as $L_{c}=c\left(L_{c}\right) \Gamma$. Substituting Eqs. (17), (20) and (21) into Eq. (29), we obtain

$$
\Gamma=\left(1+\frac{1}{\nu}\right) \frac{\Delta \gamma}{\tilde{p}\left(\tilde{U}_{a}-\tilde{U}_{i}\right)}=\frac{\tilde{K}^{\frac{1}{\nu}} \Delta \gamma}{g_{\nu}\left(\frac{\tilde{G}}{K}\right) \tilde{p}^{1+\frac{1}{\nu}}},
$$

where

$$
g_{\nu}(x) \equiv\left(\frac{2}{\nu+1}\right)^{1+\frac{1}{\nu}} \frac{\nu}{2}\left\{1-(1+x)^{-\frac{1}{\nu}}\right\}
$$




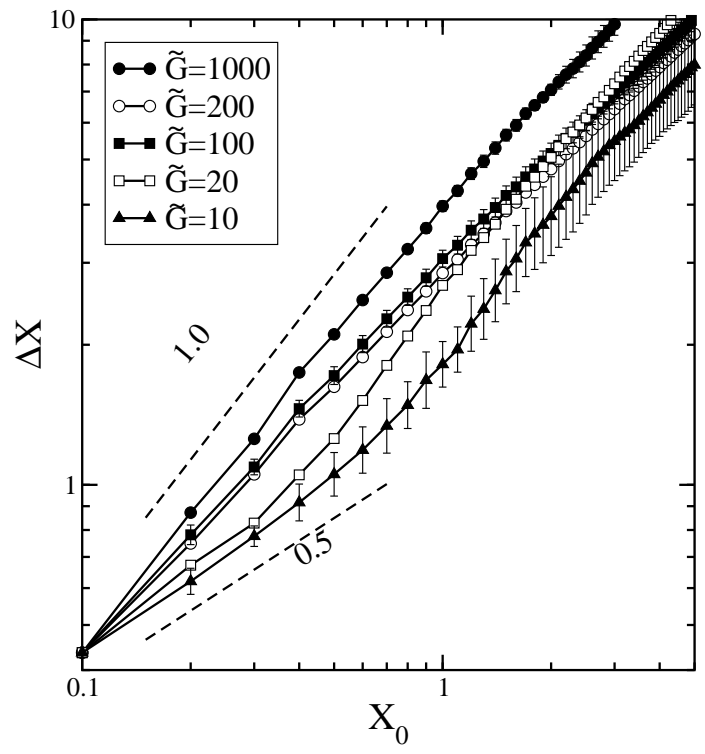

FIG. 5. Dependence of $\Delta X$ on $X_{0}$ for $\tilde{K}=1000$ and $\Delta \gamma=$ 0.001 . The results for each value of $\tilde{G}$ were calculated from the average of $X_{1}$ over eight numerical simulations on a lattice with $N_{x}=N_{y}=40$. The error bars for the plots with $\tilde{G}=20$ and 200 are omitted for clarity.

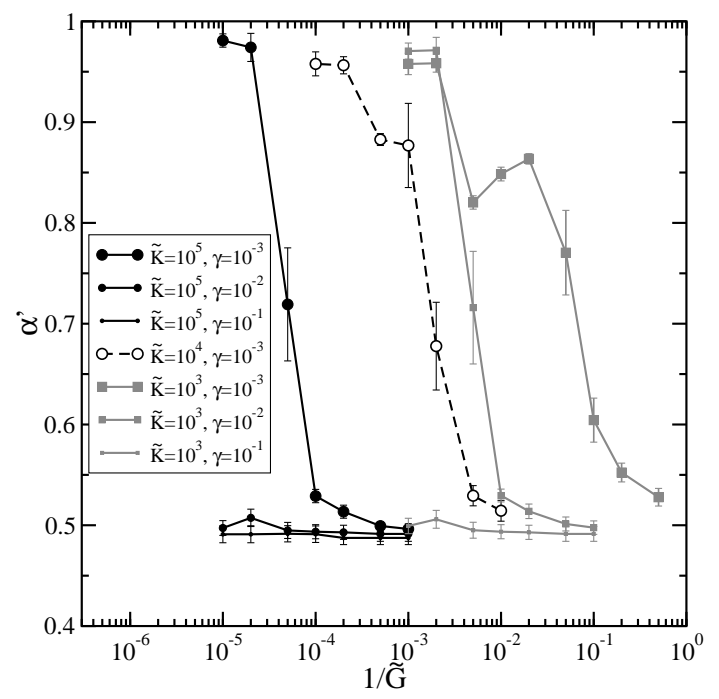

FIG. 6. Dependence of $\alpha^{\prime}$ on $1 / \tilde{G}$ for various $\tilde{K}$ and $\Delta \gamma$. These results were obtained by applying a least-square method to a log-log plot of $\Delta X$ and $X_{0}$ for $X_{0}<1$ to calculate $\alpha^{\prime}$.

The quantity $g_{\nu}(x)$ is an increasing function of $x$ and is approximately proportional to $x / 2$ for $x \ll 1$. From Eq. (18), we find $\tilde{p} \simeq 1$ for $\tilde{K} \gg 1$ and $\Delta \gamma \ll 1$.

\section{B. Verification in numerical simulations}

In order to verify the criterion appearing in Eq. (28) on the basis of our numerical simulations, it is necessary to

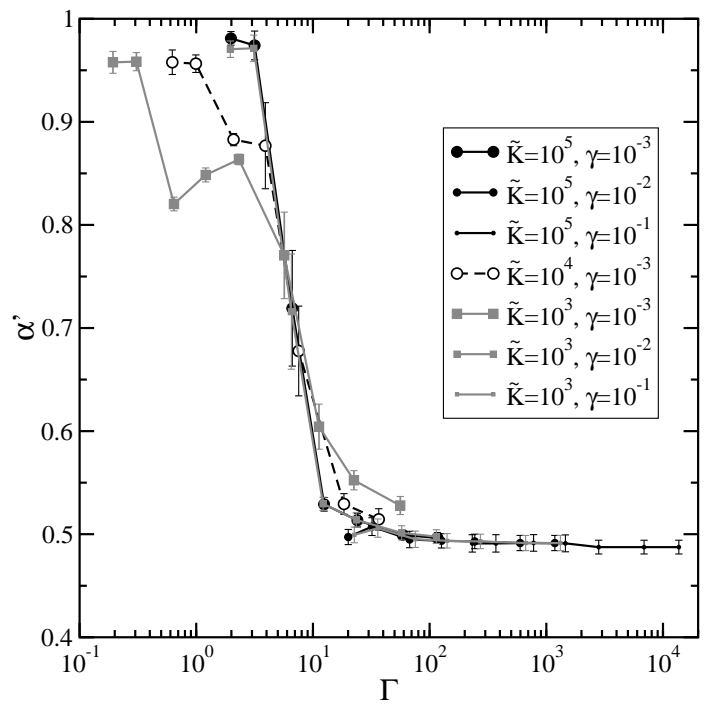

FIG. 7. Dependence of $\alpha^{\prime}$ on $\Gamma$. The data in Fig. 6] are replotted with respect to $\Gamma$, defined in Eq. (30).

quantify the transition from the invasion of the conventional IP model to cracklike invasion in the initial stage. We calculated the quantities

$$
X_{k} \equiv \frac{1}{N_{y}} \sum_{m=1}^{M} x_{m}^{k} \phi_{m}
$$

for $k=0$ and 1 and investigated the dependence of $\Delta X \equiv$ $\sqrt{2 X_{1}-X_{0}^{2}}$ on $X_{0}$.

The conventional IP model is characterized by the selfaffine growth of a dry-wet interface. Assuming the interface to be a single-valued function $x=h(y)$ in the initial stages, the standard deviation $\sqrt{\left\langle(h-\langle h\rangle)^{2}\right\rangle}$ increases as a power function of the average height $\langle h\rangle$, where $\langle\cdot\rangle$ represents the average over $y$. Because $\phi_{m}=1$ for $0 \leq x_{m}<h(y)$, the standard deviation corresponds to $\Delta X$, as we have $X_{1} \simeq\left\langle\int_{0}^{h} d x x\right\rangle=\left\langle h^{2}\right\rangle / 2$ and $X_{0} \simeq\langle h\rangle$. Contrastingly, in a cracklike process, $\sqrt{X_{1}}$ and, hence, $\Delta X$ are proportional to $X_{0}$, because in such a process, a one-dimensional dry region develops. Figure 5 displays a typical dependence of $\Delta X$ on $X_{0}$. As seen there, the invasion changes from that described by the conventional IP model to cracklike invasion as $\tilde{G}$ increases. The exponent $\alpha^{\prime}$, defined by $\Delta X \propto X_{0}^{\alpha^{\prime}}$ at $X_{0} \simeq 0$, is approximately 0.5 for the invasion of the conventional IP model and 1 for cracklike invasion.

Figure 6 displays the exponent $\alpha^{\prime}$ as a function of $1 / \tilde{G}$ for various $\tilde{K}$ and $\Delta \gamma$. The change of $\alpha^{\prime}$ from 1.0 to 0.5 corresponds to the transition of the invasion type. The data are replotted with respect to $\Gamma$ in Fig. 7. It is seen that all data approximately collapse to a single curve for large $\tilde{K}$, and the transition occurs at approximately the same value of $\Gamma$ in each case, near $\Gamma \simeq 10$. This result is consistent with the criterion appearing in Eq. (28) and indicates that $L_{c} / c\left(L_{c}\right) \simeq 10$ in our simulations. 


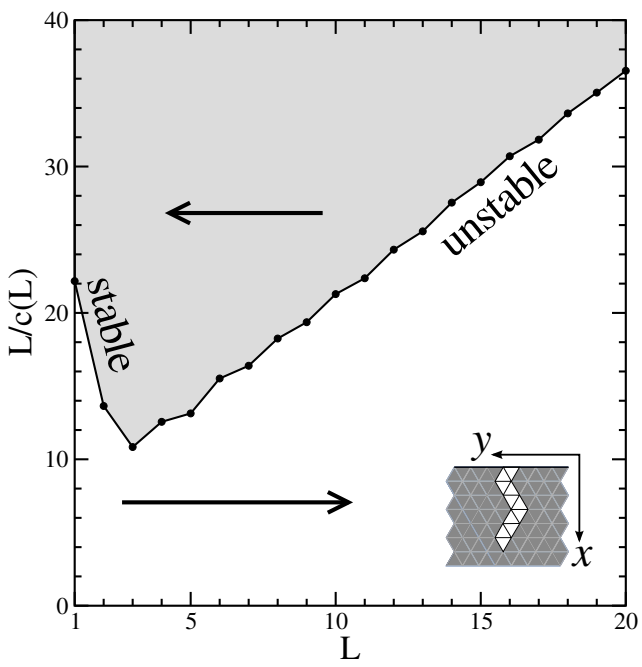

FIG. 8. $L / c(L)$ for a triangular lattice of $N_{x}=N_{y}=40$. The quantity $c(L)$ used here is the average over 100 sets of $\left\{\gamma_{m}\right\}$ created from different random seeds. The inset displays an example of a one-dimensional path with length $L=11$.

The function $c(L)$ is determined from the distribution function of $\gamma_{m}$ and the lattice properties. Investigating all one-dimensional paths of length $L$, extending from the top surface in the $x$ direction, we determined $c(L)$ from the minimum value of the sum of $\gamma_{m}$ over a path. Figure 8 displays $L / c(L)$ for the $N_{x}=N_{y}=40$ triangular lattice we used. It is seen that, because $c(L)$ increases with $L$ from $\simeq 0$ at $L=1, L / c(L)$ first decreases and then increases as $L$ increases from 1 . For the portion of this graph in which $L / c(L)$ is increasing, the value of $L$ along the curve is $L_{c}$, the crack length beyond which a crack grows unstably. For the portion of this graph in which $L / c(L)$ is decreasing, the value of $L$ along the curve corresponds to the depth of the dry region that develops following the first invasion due to heterogeneity. The minimum of $c(L) / L$ corresponds to the transition point of $\Gamma$ below which $L_{c}$ vanishes and the first invasion spontaneously induces an initial crack that develops into cracklike invasion. The value found here for $\Gamma$ is consistent with $\Gamma \simeq 10$, obtained from Fig. 7 .

The quantity $\Gamma$ generally depends on the particle size. In the original, unscaled system, $(\tilde{K}, \tilde{G})$ correspond to $(K, G) \equiv\left(p_{\gamma} / v_{w}^{\nu}\right)(\tilde{K}, \tilde{G})$. For fixed elastic properties, represented by $(K, G)$ and heterogeneity, represented by $\Delta \gamma$, we find from Eq. (4) that $\Gamma$ given in Eq. (30) with $\tilde{p} \simeq 1$ increases with the size of a particle as

$$
\Gamma \simeq v_{w} p_{\gamma}^{-\frac{1}{\nu}} \frac{\Delta \gamma K^{\frac{1}{\nu}}}{g_{\nu}\left(\frac{G}{K}\right)} \propto r^{\frac{1}{\nu}}
$$

This implies that cracking occurs only when the particle size is small. Similar results have been reported previously in some experiments [30].

\section{Discussion on the validity}

We now discuss the validity of the cracking criterion given in Eq. (28). This criterion seems valid for large $\tilde{K}$, even when shear bands appear in advance of the first crack. This is because this crack will simply follow the path of the shear band. Most of the difference in the free energy is consumed when the shear band forms, because a shear band enables compression in the surrounding region, due to the large strain it creates. By contrast, cracks tend to become wide for sufficiently soft materials. This is due to the fact that if a one-dimensional crack were to develop in such materials, the large expansion at a crack tip would cause additional dissipation, larger than $O\left((\Delta \gamma)^{2}\right)$, in Eq. (26). The dependence of $\alpha^{\prime}$ on $\Gamma$ seen in Fig. 7 deviates from the master curve for small $\tilde{K}$, because we assumed a crack to be a one-dimensional region when we derived our criterion. In order to generalize our criterion to be applicable to such cases, we need to evaluate elastic strains at the tip of a blunt crack.

Cracking in pastelike materials is often accompanied by large plastic relaxation [11] although we have ignored plasticity to elucidate the Griffith criterion in this paper. Plastic deformation would increase additional dissipation significantly. If plastic deformation occurs locally in the vicinities of crack tips, our criterion could be extended to include dissipation energy in the same manner as in the standard fracture mechanics [42]. For pastelike materials, however, we should note that plastic deformation may occur globally by increasing negative pore pressures 8] and affect crack directions through the memory effects 4 , 12.

We have investigated the first crack appearing in a uniform layer without initial cracks or flaws, except microscopic heterogeneity of drying properties. As $\Gamma$ does not depend on the layer thickness $H$ in Eq. (33), our results appear to contradict the existence of the critical cracking thickness. However, if the layer contains an initial crack or a macroscopic air-invaded region initially, it can develop and divide the system at smaller values of $\tilde{p}$. When $\tilde{p}$ is very small, the last term in Eq. (24) can be approximated as $L \Delta V\left\{-\gamma_{D}(L)\right\}$, and the dissipation term can be ignored. In this case, the cracking condition can be written

$$
L\left\{\tilde{f}_{e}\left(\tilde{\boldsymbol{U}}_{a}\right)-\tilde{f}_{e}\left(\tilde{\boldsymbol{U}}_{i}\right)+\tilde{p}\left(\tilde{U}_{a}-\tilde{U}_{i}\right)\right\} \gtrsim \gamma_{D}(\infty),
$$

because $\gamma_{D}(L)$ is approximately constant for $L \gg 1$. Assuming $L$ to be the layer thickness, $H$, this condition gives

$$
\tilde{p} \simeq\left(\frac{\tilde{K}^{\frac{1}{\nu}} \gamma_{D}(\infty)}{g_{\nu}\left(\frac{\tilde{G}}{\tilde{K}}\right) H}\right)^{\frac{\nu}{\nu+1}}
$$

as the smallest value of the pressure for which cracking can occur. For the original, unscaled system, we have $p \propto H^{-\frac{\nu}{\nu+1}}$, and $p$ does not depend on $r$, because $H$ 
is scaled by the unit of length, which is proportional to $r$. These dependencies are consistent with the theoretical and experimental results obtained in previous research [13, 15, 17, 43].

\section{CONCLUSIONS}

We proposed an invasion percolation model for a cohesionless elastic material to investigate drying processes of pastelike materials. We derived a cracking condition that applies to cohesionless porous systems taking the same form as the Griffith criterion, after eliminating local dissipation accompanied by air invasion. The Griffith energy corresponds to an additional energy required for drying, not the surface energy of the liquid-air interface itself. We find that cracklike air invasion occurs for soft materials with larger rigidity and less heterogeneity in the properties characterizing the drying process. Also, this criterion explains why cracking does not occur for systems composed of large particles.

For systems in which there is fast drying or large deformation, the cracking condition will differ from that derived here, because in such situations, there are complications that were not accounted for in the present work. Specifically, in the case of fast drying, the pore pressure will become nonuniform, while in the case of larger deformation, plastic deformation will appear. While it is important to elucidate such phenomena, these problems are beyond the scope of the present work.

\section{ACKNOWLEDGMENTS}

We thank H. Ito for her contribution to early numerical results. The author also acknowledges A. Nakahara, Ooshida T, T. Mizuguchi, S. Tarafder, T. Dutta, and C. Urabe for useful discussions and G. C. Paquette for valuable comments. This research was supported by two Grants-in-Aid for Scientific Research (Grant No. KAKENHI C 23540452 and Grant No. KAKENHI B 22340112) from JSPS, Japan.

\section{Appendix A: Equilibrium conditions}

Let us consider thermal equilibrium states of a paste system for given $(T, P, h)$, where the relative humidity, $h$, is given by $h \equiv P_{v} / P_{v}^{*}$, where $P_{v}$ is the vapor pressure and $P_{v}^{*}$ is its saturated value. We assume that both the particles and the liquid composing the system are incompressible and that the vapor is an ideal gas, for simplicity.

A condition of mechanical equilibrium, Laplace's law, states that the liquid pressure, $P_{l}$, and the atmospheric pressure, $P$, are related as

$$
p \equiv P-P_{l}=\gamma_{l a} \kappa .
$$

Here $\gamma_{l a}$ is the surface tension of the liquid-air interface and $\kappa$ is the mean curvature, which is constant everywhere on the liquid-air interface in an equilibrium state.

The negative pore pressure $p$ is determined from $h$ by the Kelvin condition,

$$
p=-k_{B} T \rho_{l} \log h,
$$

for an ideal gas, where $k_{B}$ is Boltzmann's constant and $\rho_{l} \equiv N_{l} / V_{l}$ is the number density of liquid molecules. This equation is derived from the chemical equilibrium condition $\mu_{l}\left(T, P_{l}\right)=\mu_{v}\left(T, P_{v}\right)$ and the condition for a flat interface, $\mu_{l}(T, P)=\mu_{v}\left(T, P_{v}^{*}\right)$, which provides the definition of $P_{v}^{*}$. In the derivation of Eq. (A2), the relations $\partial \mu_{l} / \partial P_{l}=1 / \rho_{l}$ and $\mu_{v}=k_{B} T \log P_{v}+$ const. have been used.

Another mechanical equilibrium condition, YoungDupré's law,

$$
\gamma_{l a} \cos \theta=\gamma_{s a}-\gamma_{s l}
$$

holds at the contact points of the liquid-air interface and the surfaces of solid particles. Here, $\theta$ is the contact angle. The interface energy of the paste is the sum of the surface energies of the liquid-air, solid-air, and solid-liquid interfaces. Explicitly, we have $F_{i}=$ $\gamma_{l a} A_{l a}+\gamma_{s a} A_{s a}+\gamma_{s l} A_{s l}$, where $A_{m n}$ and $\gamma_{m n}$ are the surface area and the surface tension of the interface indicated by their indices, respectively. As the total area of the solid surfaces, $A_{s a}+A_{s l}$, is approximately constant, $F_{i}$ can be written as

$$
F_{i}=\gamma_{l a} A+\text { const. }
$$

where $A \equiv A_{l a}+\cos \theta A_{s a}$, after substituting Eq. (A3). The invasion of air causes $A$ and thus $F_{i}$ to increase.

\section{Appendix B: Isotropic compressive states}

The free energy $\tilde{J}$ of a wet cell is minimal with respect to $\tilde{u}_{\alpha \beta}$ in the isotropic compressive state. For Eqs. (13) and (14), $\tilde{J}$ depends on $\tilde{u}_{\alpha \beta}$ as

$\tilde{f}_{e}+\tilde{p} \tilde{u}_{\alpha \alpha}=\frac{1}{2}(\tilde{K}-\tilde{G})\left(-\tilde{u}_{\alpha \alpha}\right)^{\nu+1}+\tilde{G}\left(-\tilde{u}_{\eta \eta}\right)^{\nu-1} \tilde{u}_{\alpha \beta}^{2}+\tilde{p} \tilde{u}_{\alpha \alpha}$.

When the strain tensor deviates from that of an isotropic state by $U_{\alpha \beta}$, taking the form $\tilde{u}_{\alpha \beta}=\tilde{U}_{i}\left(\delta_{\alpha \beta} / 2+U_{\alpha \beta}\right)$, this equation can be approximated to second order in $U_{\alpha \beta}$ as

$$
\begin{aligned}
\tilde{f}_{e} & +\tilde{p} \tilde{u}_{\alpha \alpha} \\
& \simeq \text { const. }+\left\{\tilde{p}-\frac{\nu+1}{2}\left(-\tilde{U}_{i}\right)^{\nu} \tilde{K}\right\} \tilde{U}_{i} U_{\alpha \alpha} \\
& +\left(-\tilde{U}_{i}\right)^{\nu+1}\left\{\frac{(\nu+1) \nu \tilde{K}-2 \tilde{G}}{4} U_{\alpha \alpha}^{2}+\tilde{G} U_{\alpha \beta}^{2}\right\}
\end{aligned}
$$

Applying the condition that this quantity be minimal gives Eq. (20) and $\tilde{K}, \tilde{G}>0$. The bulk modulus 
and rigidity in $2 \mathrm{D}$ linear elasticity are $\tilde{K}^{\prime} \equiv(\nu+$ 1) $\nu\left(-\tilde{U}_{i}\right)^{\nu-1} \tilde{K} / 2$ and $\tilde{G}^{\prime} \equiv\left(-\tilde{U}_{i}\right)^{\nu-1} \tilde{G}$, respectively, for the isotropic compressive state. Poisson's ratio is given by $\left(\tilde{K}^{\prime}-\tilde{G}^{\prime}\right) /\left(\tilde{K}^{\prime}+\tilde{G}^{\prime}\right)$.

\section{Appendix C: Perturbation from a uniaxial compressive state}

A wet cell in contact with a dry-wet interface has the same stress conditions, $\tilde{\sigma}_{x x}=\tilde{\sigma}_{x y}=0$, as the unixaial compressive state, described by $\tilde{\boldsymbol{U}}_{a}$ given in Eq. (16), where the $x$ axis is perpendicular to the interface, while the stress $\tilde{\sigma}_{y y}$ depends on the volumetric strain, $\tilde{u}_{\alpha \alpha}$.

The elastic energy is $\tilde{f}_{e}(\tilde{\boldsymbol{U}})=\tilde{f}_{e}\left(\tilde{\boldsymbol{U}}_{a}\right)+\tilde{\sigma}_{e \alpha \beta}\left(\tilde{\boldsymbol{U}}_{a}\right) \delta \tilde{u}_{\alpha \beta}+$ $O\left(\delta \tilde{\boldsymbol{U}}^{2}\right)$ for $\tilde{\boldsymbol{U}} \equiv \tilde{\boldsymbol{U}}_{a}+\delta \tilde{\boldsymbol{U}}$, where $\tilde{\sigma}_{e \alpha \beta} \equiv \partial \tilde{f}_{e} / \partial \tilde{u}_{\alpha \beta}=$ $\tilde{\sigma}_{\alpha \beta}-\tilde{p} \delta_{\alpha \beta}$. The first-order term can be rewritten as

$$
\begin{aligned}
\tilde{\sigma}_{e \alpha \beta} \delta \tilde{u}_{\alpha \beta} & =\frac{1}{\nu} \tilde{u}_{\alpha \beta} \delta \tilde{\sigma}_{e \alpha \beta} \\
& =\frac{1}{\nu}\left(\tilde{u}_{x x} \delta \tilde{\sigma}_{e x x}+\tilde{u}_{x y} \delta \tilde{\sigma}_{e x y}+\tilde{u}_{y y} \delta \tilde{\sigma}_{e y y}\right)(\mathrm{C} 1)
\end{aligned}
$$

because $\tilde{f}_{e}=\tilde{\sigma}_{e \alpha \beta} \tilde{u}_{\alpha \beta} /(\nu+1)$ for the homogeneous function given in Eq. (14) and $d \tilde{f}_{e}=\tilde{\sigma}_{e \alpha \beta} d \tilde{u}_{\alpha \beta}=$ $\left(\tilde{\sigma}_{e \alpha \beta} d \tilde{u}_{\alpha \beta}+d \tilde{\sigma}_{e \alpha \beta} \tilde{u}_{\alpha \beta}\right) /(1+\nu)$. Equation (C1) vanishes, because $\delta \tilde{\sigma}_{e x x}=\delta \tilde{\sigma}_{e x y}=0$ for fixed $\tilde{p}$, due to the stress conditions and $\tilde{u}_{y y}\left(\tilde{\boldsymbol{U}}_{a}\right)=0$. Thus, we have $\tilde{f}_{e}(\tilde{\boldsymbol{U}})-\tilde{f}_{e}\left(\tilde{\boldsymbol{U}}_{a}\right)=O\left(\delta \tilde{\boldsymbol{U}}^{2}\right)$ and

$$
\tilde{\sigma}_{e \alpha \beta} \delta \tilde{u}_{\alpha \beta}=-\tilde{p} \delta \tilde{u}_{x x}+\tilde{\sigma}_{e y y} \delta \tilde{u}_{y y}=0 .
$$

The quantity $\delta \tilde{\boldsymbol{U}}$ is determined from Eq. (C2) and $\delta \tilde{\sigma}_{x y}=$ 0 for a given $\delta \tilde{u}_{\alpha \alpha}=\delta \tilde{u}_{x x}+\delta \tilde{u}_{y y}$, and thus we find

$$
\tilde{f}_{e}(\tilde{\boldsymbol{U}})-\tilde{f}_{e}\left(\boldsymbol{U}_{a}\right)=O\left(\left(\delta \tilde{u}_{\alpha \alpha}\right)^{2}\right) .
$$

[1] L. Pauchard, M. Adda-Bedia, C. Allain, and Y. Couder, Phys. Rev. E 67, 027103 (2003).

[2] S. Bohn, L. Pauchard, and Y. Couder, Phys. Rev. E 71, 046214 (2005).

[3] S. Bohn, J. Platkiewicz, B. Andreotti, M. Adda-Bedia, and Y. Couder, Phys. Rev. E 71, 046215 (2005).

[4] A. Nakahara and Y. Matsuo, Phys. Rev. E 74, 045102(R) (2006).

[5] D. Mal, S. Sinha, T. R. Middya, and S. Tarafdar, Appl. Clay Sci. 39, 106 (2008).

[6] L. Pauchard, B. Abou, and K. Sekimoto, Langmuir 25, 6672 (2009).

[7] S. Kitsunezaki, J. Phys. Soc. Jpn. 78, 064801 (2009).

[8] S. Kitsunezaki, J. Phys. Soc. Jpn. 79, 124802 (2010).

[9] L. Goehring, R. Conroy, A. Akhter, W. J. Clegg, and A. F. Routh, Soft Matter 6, 3562 (2010).

[10] L. Goehring, W. J. Clegg, and A. F. Routh, Langmuir 26, 9269 (2010).

[11] L. Goehring, W. J. Clegg, and A. F. Routh, Phys. Rev. Lett. 110, 024301 (2013).

[12] A. Nakahara, Y. Shinohara, and Y. Matsuo, J. Phys. : Conf. Ser. 319, 012014 (2011).

[13] A. F. Routh and W. B. Russel, Langmuir 15, 7762 (1999).

[14] M. S. Tirumkudulu and W. B. Russel, Langmuir 21, 4938 (2005).

[15] K. B. Singh and M. S. Tirumkudulu, Phys. Rev. Lett. 98, 218302 (2007).

[16] W. B. Russel, N. Wu, and W. Man, Langmuir 24, 1721 (2008).

[17] W. Man and W. B. Russel, Phys. Rev. Lett. 100, 198302 (2008).

[18] D. M. Wood, Soil Behaviour and Critical State Soil Mechanics (Cambridge University Press, New York, 1990).

[19] M. Otsuki, Phys. Rev. E 72, 046115 (2005).

[20] Ooshida Takeshi, Phys. Rev. E 77, 061501 (2008).

[21] D. Wilkinson and J. F. Willemsen, J. Phys. A 16, 3365
(1983).

[22] D. Wilkinson, Phys. Rev. A 30, 520 (1984).

[23] D. Wilkinson, Phys. Rev. A 34, 1380 (1986).

[24] C. Du, B. Xu, Y. C. Yortsos, M. Chaouche, N. Rakotomalala, and D. Salin, Phys. Rev. Lett. 74, 694 (1995).

[25] Y. C. Yortsos, B. Xu, and D. Salin, Phys. Rev. Lett. 79, 4581 (1997).

[26] P. Meakin, G. Wagner, A. Vedvik, H. Amundsen, J. Feder, and T. Jøssang, Mar. Petrol. Geol. 17, 777 (2000).

[27] G. Løvoll, Y. Méheust, K. J. Måløya, E. Aker, and J. Schmittbuhl, Energy 30, 861 (2005).

[28] Y. Yamazaki, S. Komura, and K. Suganuma, J. Phys. Soc. Jpn. 75, 043001 (2006).

[29] H. Nakanishi, R. Yamamoto, Y. Hayase, and N. Mitarai, J. Phys. Soc. Jpn. 76, 024003 (2007).

[30] L. Xu, S. Davies, A. B. Schofield, and D. A. Weitz, Phys. Rev. Lett. 101, 094502 (2008).

[31] N. Shokri and D. Or, J. Colloid Interface Sci. 391, 135 (2013).

[32] Strong cohesion is often formed among constituent particles, typically after drying [44]. In such cases, cracking has common properties with typical brittle fracture in contrast to that in capillary states [7].

[33] L. Goehring, Phys. Rev. E 80, 036116 (2009).

[34] Y. Jiang and M. Liu, Eur. Phys. J. E 22, 255 (2007).

[35] S. Kitsunezaki, Phys. Rev. E 60, 6449 (1999).

[36] W. H. Press, S. A. Teukolsky, W. T. Vetterling, and B. P. Flannery, Numerical Recipes in $\mathrm{C}++$, 2nd ed. (Cambridge University Press, New York, 2002).

[37] B. K. Chakrabarti and L. G. Benguigui, Statistical Physics of Fracture and Breakdown in Disordered Systems (Oxford University Press, New York, 1997).

[38] A. Shekhawat, S. Zapperi, and J. P. Sethna, arXiv:1210.0989 (2012).

[39] R. Holtzman and R. Juanes, Phys. Rev. E 82, 046305 
(2010)

[40] R. Holtzman, M. L. Szulczewski, and R. Juanes, Phys. Rev. Lett. 108, 264504 (2012).

[41] S. Kitsunezaki, Adv. Powder Technol. 22, 311 (2011).
[42] B. Lawn, Fracture of Brittle Solids, 2nd ed. (Cambridge University Press, Cambridge, 1993).

[43] J. L. Beuth Jr, Int. J. Solids Structures 29, 1657 (1992).

[44] K. Kendall, N. M. Alford, and J. D. Birchall, Proc. R. Soc. Lond. A 412, 269 (1987). 